\title{
CONDIÇÃO DE SAÚDE FUNCIONAL E CAPACIDADE PARA O TRABALHO DE AGROPECUARISTAS DE NOVA VENEZA/SC
}

DOI: http://dx.doi.org/10.18616/prat12

\begin{abstract}
Larissa Damiani Romagna UNESC - Universidade do Extremo Sul Catarinense lari-dr@hotmail.com

Tainara Tramontin UNESC - Universidade do Extremo Sul Catarinense tai_tramontin@hotmail.com

Willians Cassiano Longen UNESC - Universidade do Extremo Sul Catarinense wcl@unesc.net
\end{abstract}




\section{INTRODUÇÃO}

O interesse pela saúde de populações que habitam regiões rurais tem se elevado em todo o mundo, buscando serviços de saúde para o seu atendimento (TECKLE, HANNAFORD; SUTTON, 2012; MOREIRA et al., 2015). Este interesse ocorre por conta de que o setor agrícola é um dos três setores mais perigosos do mundo do trabalho, onde os trabalhadores rurais estão expostos a uma variedade de fatores que afetam sua segurança e saúde (KOLSTRUP, 2012; LUNNER-KOLSTRUP; SSALI, 2016).

O tipo e a natureza das tarefas, incluindo as subdivisões de trabalho no setor agrícola, geralmente, exigem que os trabalhadores adotem posturas incômodas. Essas posturas incluem inclinar-se para frente e para o lado, ajoelhar, rastejar e torcer, o que pode induzir ao estresse traumático e aos danos físicos (TAGHAVI et al., 2016). Os distúrbios músculo-esqueléticos relacionados ao trabalho são descritos como uma das principais doenças relacionadas aos trabalhadores do setor agrícola (TAGHAVI et al., 2016; WALKER-BONE; PALMER, 2002; MCMILLAN, 2010). Inicialmente, essas lesões podem surgir através de um quadro álgico, podendo evoluir para problemas maiores, como é o caso das Lesões por Esforços Repetitivos (LER) / Distúrbios Osteomusculares Relacionados ao Trabalho (DORT) (MCMILLAN, 2010).

Estes fenômenos são resultados de uma combinação da sobrecarga do sistema osteomuscular, que pode ocorrer pelo excesso do uso de determinados grupos musculares, principalmente, quando a respectiva atividade demanda de esforços e resistências contra a ação da gravidade (BACHIEGA, 2009).

As excessivas demandas físicas do trabalho são as principais causas que comumente afetam os trabalhadores rurais, sendo que as LER/DORT acometem de forma significativa esses trabalhadores, gerando dor na região lombar (DA SILVA, 2017; ALVES; GUIMARÃES, 2012). Os movimentos de elevação de tronco, flexão e extensão de forma prolongada e repetida da coluna, além do transporte de cargas pesadas são fatores de risco para o surgimento de lesões e processos álgicos (DA SILVA, 2017; FATHALLAH, 2010). Estudos 
afirmam que a lombalgia é o sintoma musculoesquelético mais frequente entre os agricultores, com uma prevalência vitalícia de 75\% (BALAGUE et al., 2012; OSBORNE et al., 2012; KANG et al., 2017).

O processo de execução do trabalho e a forma em que é realizado, considerando o levantamento de peso, movimentos bruscos de flexão e má postura durante as atividades levam os trabalhadores rurais a desenvolverem sintomas dolorosos (SARAH et al., 2011; ROCHA et al., 2014). Esses sintomas surgem de acordo com as tarefas executadas, como é o caso das atividades manuais, estando inclusos o plantio, colheita, inspeção e embalagem dos produtos, poda, carregamento, transporte de mercadorias e uso de produtos químicos. Essas condições podem acarretar diversos malefícios a saúde como fadiga generalizada, transtornos traumáticos cumulativos, contraturas musculares, dores e lesões na coluna cervical e lombar, dores nos membros superiores e inferiores, dores articulares e musculoesqueléticas, além de lesões de mão e punho (VILLAREJO et al., 2010; ROCHA et al., 2014).

O objetivo do presente estudo foi analisar a condição de saúde funcional e de capacidade para o trabalho de trabalhadores rurais, bem como a identificação de sintomas musculoesqueléticos e o índice de capacidade para o trabalho. Da mesma forma, foram levantados os segmentos corporais mais acometidos e a presença de incapacidade física subclassificada em leve, moderada e intensa. Além disso, foi verificada a percepção subjetiva de esforço relacionada às atividades mais executadas de acordo com o tipo de trabalho.

\section{MÉTODOS}

Esta pesquisa é uma abordagem transversal e quantitativa. Foi realizada no Sindicato dos Trabalhadores Rurais do município de Nova Veneza-SC entre os meses de agosto e outubro de 2018. Foram incluídos neste estudo, somente indivíduos com idade entre 25 e 60 anos, de ambos os sexos, sendo trabalhador rural ativo. Este estudo foi aprovado pelo comitê de ética da Universidade do Extremo Sul Catarinense (UNESC) sob o parecer n. 2.718.610. 
O cálculo da amostra foi realizado por meio do programa Cálculo Amostral - calculadora on-line (SANTOS, 2018). Com base nos dados do Sindicato dos Trabalhadores Rurais da região de Nova Veneza, o número de trabalhadores agropecuários cadastrados no município foi de 908, representando a população da pesquisa. A amostra alvo foi de 45 trabalhadores, recrutados de forma aleatória.

Foi aplicado o Questionário Sociodemográfico composto por oito questões de múltipla escolha, o qual objetivou a análise da sintomatologia relacionada a distúrbios osteomusculares nos trabalhadores rurais, de acordo com o setor de envolvimento. Dessa maneira, possibilitou a coleta de dados pessoais e de dados relacionados ao trabalho, na qual o indivíduo respondeu às alternativas que melhor definem suas condições de trabalho e saúde.

Para quantificar o esforço realizado no trabalho do participante, recorreu-se a Escala Psicofísica CR-10 De Borg, apresentada por meio de uma tabela (LIGEIRO, 2010). Empregou-se o Nordic Musculoskeletal Questionnaire (NMQ) para análise e detecção dos sintomas musculoesqueléticos, composto em 28 questões de múltipla escolha (DICKINSON et al., 1992; LÓPEZARAGÓN et al., 2017).

Para análise da dor do voluntário, utilizou-se a Escala Visual Analógica (EVA), a qual foi apresentada por meio de uma escala referencial com cores, números e caretas determinando a ausência de dor até a dor máxima (HERR et al., 2010; PESONEN, 2018; CUSTÓDIA, 2015).

O Oswestry Low Back Pain Disability Questionnaire (ODQ) foi utilizado com o intuito de avaliar disfunções nos participantes com lombalgias por meio de uma análise das atividades de vida diária. Questões relacionadas aos membros superiores também foram incluídas no questionário, levando em consideração os níveis de dificuldade durante as atividades de vida diária (FAIRBANK, 1980; WALSH, 2005; MASSELLI et al., 2007).

O Índice de Capacidade para o Trabalho (ICT) foi aplicado para classificar a compreensão do indivíduo em relação ao quão bem os trabalhadores estão no momento atual e como estará no futuro. Bem como, explorou o quão 
bem eles podem realizar o trabalho, em função das condições impostas, de seu estado de saúde e capacidades físicas e mentais (SILVA et al., 2013).

Os dados coletados foram organizados e analisados com auxílio do programa IBM Statistical Package for the Social Sciencies (SPSS) versão 23.0. As variáveis quantitativas foram expressas por meio de média e desvio padrão. As variáveis qualitativas foram expressas por meio de frequência e porcentagem.

\section{RESULTADOS}

A média de idade dos 45 trabalhadores agropecuários foi 51,24 $( \pm 7,81)$ anos, sendo $71,1 \%$ dos indivíduos do sexo masculino e $28,9 \%$ do sexo feminino. O tempo de atividade no ramo agrícola alcançou 36,69 $( \pm 12,54)$ anos, com uma jornada diária de $9,96( \pm 2,48)$ horas. Quanto à posição mais adotada durante o trabalho, $82,2 \%$ participantes responderam que sua posição predominante no dia a dia é a posição ortostática, seguido de 15,6\% que responderam a posição em sedestação e 2,2\% de cócoras. Em relação ao cansaço, $42,2 \%$ responderam sentir-se cansados no final do dia de trabalho, $22,2 \%$ exaustos, $17,8 \%$ pouco cansados, $15,6 \%$ muito cansados e $2,2 \%$ nenhum cansaço (Tabela 1).

Tabela 1 - Caracterização dos Trabalhadores Agropecuários e Dados Referentes ao Trabalho

\begin{tabular}{c|c}
\hline & Média \pm DP; $\mathbf{n}(\%) \mathbf{n}=\mathbf{4 5}$ \\
\hline \multicolumn{2}{|c}{ Sexo } \\
\hline Idade (anos) & $31,24 \pm 7,81$ \\
\hline Masculino & $13(28,9)$ \\
\hline Feminino & $36,69 \pm 12,54$ \\
\hline Horas de trabalho & $9,96 \pm 2,48$ \\
\hline \multicolumn{2}{c}{ Posição de trabalho } \\
\hline
\end{tabular}




\begin{tabular}{c|c}
\hline & Média \pm DP; $\mathbf{n}(\mathbf{\%}) \mathbf{n}=\mathbf{4 5}$ \\
\hline De pé & $37(82,2)$ \\
\hline Sentado & $7(15,6)$ \\
\hline Cócoras & $1(2,2)$ \\
\hline
\end{tabular}

\begin{tabular}{c|c}
\hline \multicolumn{2}{c}{ Cansaço } \\
\hline Nenhum cansaço & $1(2,2)$ \\
\hline Pouco cansado & $8(17,8)$ \\
\hline Cansado & $19(42,2)$ \\
\hline Muito cansado & $7(15,6)$ \\
\hline Exausto & $10(22,2)$ \\
\hline
\end{tabular}

Fonte: Dados da Pesquisa (2018).

DP-Desvio Padrão.

Para estimar a prevalência de distúrbios musculoesqueléticos relacionados ao trabalho foi utilizado o Nordic Musculoskeletal Questionnaire. O segmento mais mencionado foi a coluna lombar, envolvendo 60,0\% dos agricultores, que relataram dor, formigamento ou dormência nessa região nos últimos 12 meses; 15,6\% foram impedidos de realizar suas atividades nos últimos 12 meses por problemas nessa região, 22,2\% consultaram médicos ou fisioterapeutas nos últimos 12 meses por conta dessa condição, 15,6\% tiveram problemas na região lombar nos últimos 7 dias. Em seguida, o segmento mais citado foram os ombros, sobre os quais $26,7 \%$ manifestaram sintomas nos últimos 12 meses, 8,9\% foram impedidos de realizar atividades nos últimos 12 meses, $11,1 \%$ consultaram profissionais saúde, $11,1 \%$ apresentaram problemas nos últimos 7 dias. O terceiro segmento corporal mais mencionado foram punho e mãos, sendo que 24,4\% afirmaram sintomas nos últimos 12 meses, 2,2\% tendo impedimento para realizar atividades nos últimos 12 meses, 2,2\% consultaram profissionais da saúde nos últimos 12 meses, 4,4\% apresentaram problemas nos últimos 7 dias (Tabela 2). 
Tabela 2 - Análise de Sintomas Musculoesqueléticos Através do Nordic Musculoskeletal Questionnaire (NMQ)

\begin{tabular}{c|c|c}
\hline \multirow{3}{*}{ Pescoço } & n(\%) / n=45 \\
\cline { 2 - 3 } & Sim & Não \\
\hline Dor, formigamento ou dormência (12 meses) & $5(11,1)$ & $40(88,9)$ \\
\hline Impedido de realizar atividades (12 meses) & $0(0,0)$ & $45(100,0)$ \\
\hline Consultou profissional da saúde (12 meses) & $1(2,2)$ & $44(97,8)$ \\
\hline Teve alguma dor (7 dias) & $1(2,2)$ & $44(97,8)$ \\
\hline \multicolumn{3}{c}{ Ombros } \\
\hline Dor, formigamento ou dormência (12 meses) & $12(26,7)$ & $33(73,3)$ \\
\hline Impedido de realizar atividades (12 meses) & $4(8,9)$ & $41(91,1)$ \\
\hline Consultou profissional da saúde (12 meses) & $5(11,1)$ & $40(88,9)$ \\
\hline Teve alguma dor (7 dias) & $5(11,1)$ & $40(88,9)$ \\
\hline \multicolumn{3}{c}{ Superior das Costas } \\
\hline Dor, formigamento ou dormência (12 meses) & $2(4,4)$ & $43(95,6)$ \\
\hline Impedido de realizar atividades (12 meses) & $0(0,0)$ & $45(100,0)$ \\
\hline Consultou profissional da saúde (12 meses) & $1(2,2)$ & $44(97,8)$ \\
\hline Teve alguma dor (7 dias) & $1(2,2)$ & $44(97,8)$ \\
\hline
\end{tabular}

Cotovelo

\begin{tabular}{|c|c|c|}
\hline Dor, formigamento ou dormência (12 meses) & $6(13,3)$ & $39(86,7)$ \\
\hline Impedido de realizar atividades ( 12 meses) & $1(2,2)$ & $44(97,8)$ \\
\hline Consultou profissional da saúde (12 meses) & $1(2,2)$ & $44(97,8)$ \\
\hline Teve alguma dor (7 dias) & $2(4,4)$ & $43(95,6)$ \\
\hline \multicolumn{3}{|l|}{ Punho e Mão } \\
\hline Dor, formigamento ou dormência (12 meses) & $11(24,4)$ & $34(75,6)$ \\
\hline Impedido de realizar atividades (12 meses) & $1(2,2)$ & $44(97,8)$ \\
\hline Consultou profissional da saúde ( 12 meses) & $1(2,2)$ & $44(97,8)$ \\
\hline Teve alguma dor (7 dias) & $2(4,4)$ & $43(95,6)$ \\
\hline
\end{tabular}

Costas Inferior

\begin{tabular}{c|c|c}
\hline Dor, formigamento ou dormência (12 meses) & $27(60,0)$ & $18(40,0)$ \\
\hline Dor, formigamento ou dormência (12 meses) & $10(22,2)$ & $35(77,8)$ \\
\hline
\end{tabular}




\begin{tabular}{|c|c|c|}
\hline & \multicolumn{2}{|c|}{$n(\%) / n=45$} \\
\hline & Sim & Não \\
\hline \multicolumn{3}{|l|}{ Quadril e Coxa } \\
\hline Dor, formigamento ou dormência (12 meses) & $9(20,0)$ & $36(80,0)$ \\
\hline Impedido de realizar atividades ( 12 meses) & $1(2,2)$ & $44(97,8)$ \\
\hline Consultou profissional da saúde (12 meses) & $5(11,1)$ & $40(88,9)$ \\
\hline Teve alguma dor (7 dias) & $3(6,7)$ & $42(93,3)$ \\
\hline \multicolumn{3}{|l|}{ Joelho } \\
\hline Dor, formigamento ou dormência (12 meses) & $8(17,8)$ & $37(82,2)$ \\
\hline Impedido de realizar atividades ( 12 meses) & $2(4,4)$ & $43(95,6)$ \\
\hline Consultou profissional da saúde (12 meses) & $4(8,9)$ & $41(91,1)$ \\
\hline Teve alguma dor (7 dias) & $1(2,2)$ & $44(97,8)$ \\
\hline
\end{tabular}

Tornozelo e Pé

\begin{tabular}{c|c|c}
\hline Dor, formigamento ou dormência (12 meses) & $3(6,7)$ & $42(93,3)$ \\
\hline Impedido de realizar atividades (12 meses) & $2(4,4)$ & $43(95,6)$ \\
\hline Consultou profissional da saúde (12 meses) & $1(2,2)$ & $44(97,8)$ \\
\hline Teve alguma dor (7 dias) & $0(0,0)$ & $45(100,0)$ \\
\hline
\end{tabular}

Fonte: Dados da Pesquisa (2018).

A avaliação da intensidade da dor foi classificada pela EVA, sendo que $62,2 \%$ relataram intensidade de dor moderada, $31,1 \%$ dor intensa e $6,7 \%$ dor leve (Tabela 3).

Tabela 3 - Classificação da Intensidade da Dor com a Escala Visual Analógica (EVA)

\begin{tabular}{c|c}
\hline & $\mathbf{n}(\%) / \mathbf{n}=\mathbf{4 5}$ \\
\hline Escala Visual Analógica \\
\hline Leve & $3(6,7)$ \\
\hline Moderada & $28(62,2)$ \\
\hline Intensa & $14(31,1)$ \\
\hline
\end{tabular}

Fonte: Dados da Pesquisa (2018). 
Os resultados Oswestry Low Back Pain Disability Questionnaire (ODQ) mostraram que 97,8\% dos trabalhadores apontaram incapacidade de grau mínimo para realização das atividades de vida diária, seguido de 2,2\% que demonstraram incapacidade de grau moderado e nenhum reportou grau de incapacidade severa (Tabela 4 ).

Tabela 4 - Classificação da Incapacidade com o Oswestry Low Back Pain Disability Questionnaire (ODQ)

\begin{tabular}{c|c}
\hline & $\mathbf{n}(\%) / \mathbf{n}=45$ \\
\hline \multicolumn{2}{c}{ Grau de Incapacidade } \\
\hline Mínima (0 a 19) & $44(97,8)$ \\
\hline Moderada (20 a 39) & $1(2,2)$ \\
\hline Severa (40 a 60) & $0(0,0)$ \\
\hline
\end{tabular}

Fonte: Dados da Pesquisa (2018).

O Índice de Capacidade para o Trabalho (ICT) alcançou 40\% da amostra na qual refere capacidade moderada para o trabalho; $40 \%$ das respostas foram classificadas como capacidade boa para o trabalho e $20 \%$ como capacidade baixa, sendo que nenhum dos participantes classificou sua capacidade como ótima. De acordo com seu escore final, quanto maior a pontuação, melhor será a capacidade para o trabalho. Dessa forma, obteve-se uma média de $32,90( \pm 6,57)$, classificando a capacidade para o trabalho, no geral, como moderada (Tabela 5).

De acordo com a análise, foi identificada uma elevada média de idade de 51,24 anos, tendo predomínio do sexo masculino e elevado tempo de trabalho na agricultura, em média 35 anos. Uma pesquisa com indivíduos agropecuários coreanos também apresentou elevada média de idade, sendo 56,6 anos e prevalência do sexo feminino (KANG et al., 2017). O elevado tempo de trabalho de agricultores da cidade de Irati, Paraná, apresentou uma média um pouco inferior à encontrada neste estudo com estes trabalhadores agropecuários de município do sul catarinense (STADLER, 2016). 
No presente estudo, foi verificada uma elevada carga horária diária de trabalho, correspondendo a mais de 9 horas. Em um município do Extremo Oeste catarinense, a média de tempo utilizada para o trabalho era de 11,63 horas durante a safra (DA SILVA, 2017).

A maior exposição diária com longas jornadas às condicionantes do trabalho agropecuário torna essa população trabalhadora exposta a inúmeros riscos físicos relacionados às condições climáticas devido ao trabalho a céu aberto, como altas temperaturas, chuva e radiação solar. Estão envolvidos riscos de acidentes como traumas e incêndios, além dos riscos ergonômicos que estão associados aos movimentos repetitivos, posturas inadequadas e sobrecargas físicas. $\mathrm{O}$ esforço físico associado ao ambiente de trabalho com altas temperaturas leva a sobrecarga potencializada pelo estresse térmico (LEITE et al., 2018).

A posição ortostática teve destaque em relação ao predomínio de posturas durante as atividades de trabalho. Essa posição exige uma contração mantida de músculos envolvidos na sua manutenção, tendo como consequência a fadiga muscular, além de dores e desconfortos na coluna vertebral e membros inferiores. A manutenção da posição em ortostase exige constantemente tensões musculares causando a compressão de vasos sanguíneos. Durante a contração estática dos músculos ocorre um enrijecimento tecidual sendo que a pressão intramuscular reduz o diâmetro dos vasos prejudicando a pressão sanguínea dos músculos de modo a prejudicar o retorno venoso das extremidades (BERENGUER, 2011).

Em uma pesquisa com agropecuários de um pequeno município do sul de Minas Gerais foi identificada prevalência do trabalho em ortostase, envolvendo $86,8 \%$ dos entrevistados (MANZOLI, 2017). Isso demonstra que o homem tende a se adaptar de acordo com as exigências e necessidades empregadas no ambiente de trabalho. É importante destacar a associação entre o trabalho em pé e elevadas cargas horárias de exposição, o que expõe estes trabalhadores por maiores períodos às condicionantes da posição ortostática. Uma pesquisa relata que queixas de dores, especialmente na coluna lombar, estão associadas a atividades que demandam da posição em pé (SOUZA et al., 2017). 
Este estudo destaca que 64,4\% dos indivíduos apresentaram-se entre cansados e exaustos no final do dia de trabalho. Num estudo no município de Mendonça, estado de São Paulo, o valor percentual foi distinto, com uma porcentagem de 37,7\% envolvendo o cansaço excessivo no período pós-safra (MARTINS e LONGEN, 2017). Essa condição pode estar associada à elevada carga horária diária de trabalho e às manutenções da posição ortostática associadas às demandas físicas do exercício das atividades do trabalho agropecuário identificadas neste estudo.

O predomínio de dor lombar foi elevado entre os entrevistados de um município do Extremo Oeste Catarinense, alcançando quase a totalidade com $98,7 \%$ dos voluntários, evidenciando dor de forte intensidade em 44,3\% da amostra (SILVA et al., 2013).

No presente estudo foi identificado o predomínio de dor de intensidade moderada, alcançando $62,2 \%$, sendo que $60 \%$ tiveram dores ou desconfortos na coluna lombar nos últimos 12 meses. Esses achados sugerem cronicidade, considerando o tempo de duração da dor, sua persistência e recorrência, sugerindo envolver distúrbios osteomusculares relacionados ao trabalho. Dessa forma, as dores crônicas são as que permanecem por mais de três meses, não tendo necessariamente uma causa específica, como é o caso de lombalgias (MARTINS e LONGEN, 2017). Em relação aos impactos da lombalgia no cotidiano de vida, $15,6 \%$ foram impedidos de exercer suas atividades nos últimos 12 meses e 15,6\% apresentaram dores lombares nos últimos 7 dias.

A presença de lombalgia mostrou-se dissociada de incapacidade para o trabalho, sendo classificada em grau mínimo, representando quase que a totalidade, ao envolver $97,8 \%$ dos entrevistados. É importante destacar que a frequência de lombalgia ocorreu neste estudo envolvendo uma média de idade elevada entre os agropecuaristas, carga horária diária também elevada e presença de sintomas musculoesqueléticos predominantemente na coluna lombar. Esses dados evidenciam que a dor não é um determinante de incapacidade e os trabalhadores agrícolas continuam ativos. 
Nos entrevistados do Extremo Oeste catarinense, os resultados indicaram grau mínimo de incapacidade em 78,2\% dos trabalhadores e predomínio de dor de forte intensidade, correspondendo a 44,3\%, o que consequentemente levaria ao impedimento das atividades (DA SILVA, 2017).

No presente estudo, foi evidenciado que a maioria dos participantes foi classificada com escores equivalentes à incapacidade mínima para o trabalho em relação aos elevados índices de dores na coluna lombar. Dessa maneira, os resultados apontaram que a capacidade para o trabalho destes trabalhadores foi classificada entre boa e moderada. Isso demonstra que os sintomas álgicos não levam à incapacidade por si só, envolvendo uma maior complexidade do que somente a dor lombar. É importante destacar que especialmente no tocante aos Distúrbios Osteomusculares Relacionados ao Trabalho, vários são os fatores contributivos envolvidos nas condicionantes do trabalho (LONGEN et al., 2018).

\section{CONCLUSÃO}

O trabalho agropecuário apresenta risco potencial de distúrbios musculoesqueléticos, devido às suas particularidades que colocam a pessoa exposta a riscos diversificados e concomitantes, principalmente os ergonômicos. A lombalgia crônica foi evidenciada como um distúrbio frequente nessa população trabalhadora. Chamou a atenção neste estudo o fato de que os sintomas álgicos não determinaram condições incapacitantes para o trabalho, isso apesar da existência de outros fatores que poderiam impactar no desempenho dos trabalhadores a exemplo da percepção subjetiva de esforço das atividades agropecuárias. As condições de trabalho envolvidas podem favorecer o desenvolvimento de sintomas musculoesqueléticos envolvendo esta população trabalhadora que se mostra resiliente e se mantém laboralmente ativa. Parte destes achados pode ser explicada por uma adaptação ao desconforto, pela cronificação dos sintomas e pelas necessidades imperativas de realização do trabalho. Ficou patente a demanda de realização de estudos junto ao trabalhador agropecuário na melhor compreensão dos fenômenos que envolvem sua condição funcional. 


\section{REFERÊNCIAS}

ALVES, R. A.; GUIMARÃES, M. C. De que sofrem os Trabalhadores Rurais? Análise dos Principais Motivos de Acidentes e Adoecimentos nas Atividades Rurais. Informe Gepec. 2012; 16 (2): 39-56.

BACHIEGA, J. C. Sintomas de distúrbios osteomusculares relacionados à atividade de cirurgiões-dentistas brasileiros [dissertação na Internet]. São Paulo (SP): Universidade Nove de Julho. 2009.

BALAGUE, F.; MANNION, A. F.; PELLISE, F; CEDRASCHI, C. Non-Specific Low Back Pain. Lancet. 2012; n. 379, p. 482-491.

BERENGUER, F. A.; SILVA, D. A. L.; CARVALHO, C. C. Influência da posição ortostática na ocorrência de sintomas e sinais clínicos de venopatias de membros inferiores em trabalhadores de uma gráfica na cidade de Recife-PE. 2011; v. 36, n. 123, p. 153-161.

BERNAARDS, C. M.; BOSMANS, J. E.; HILDEBRANDT, V. H.; VAN TULDER, M. W.; HEYMANS, M. W. The Cost-Effectiveness of a Lifestyle Physical Activity Intervention in Addition to a Work Style Intervention on Recovery from Neck and Upper Limb Symptoms and Pain Reduction in Computer Workers. Occup Environ Med. 2010; n. 68, p. 265-272.

CUSTÓDIA, A. C. E.; MAIA, F. O. M.; SILVA, R. C. G. Escalas de Avaliação da Dor em Pacientes Idosos com Demência. Revista Dor. 2015; n. 16, p. 4.

DA SILVA, M. R.; FERRETI, F.; LUTINSKI, J. A. Dor Lombar, Flexibilidade Muscular e Relação com o Nível de Atividade Física de Trabalhadores Rurais. Saúde em Debate. 2017; n. 41, p. 112.

DICKINSON, C. E.; CAMPION, K., FOSTER, A. F.; NEWMAN, S. J.; O'ROURKE, A. M.; THOMAS, P. G. Questionnaire Development: an Examination of the Nordic Musculoskeletal Questionnaire. Applied Ergonomics.1992; n. 23, p. 197-201. 
FAIRBANK, J. C.; COUPER, J.; DAVIES, J. B.; O'BRIEN, J. P. The OswestryLow Back Pain Disability Questionnaire. Physiotherapy. 1980; v. 66, n. 8, p. 271.

FATHALLAH, F. A. Musculoskeletal Disorders in Labor-Intensive Agriculture. Appl Ergon. 2010; v. 46, n. 6, p. 738-743.

HERR, K.; BURSCH, H.; ERSEK, M.; MILLER, L. L.; SWAFFORD, K. Use of Pain-Behavioral Assessment Tools in the Nursing Home: expert consensus recommendations for practice. J Gerontol Nurs. 2010; v. 36, n. 3, p. 18-29.

KANG, E. K.; PARK, H. W.; KIM, S. H.; BAEK, S. Clinical Usefulness of X-Ray Findings for Nonspecific Low Back Pain in Korean Farmers: farm study. Annals of Rehabilitation Medicine. 2017; n. 41, p. 808-815.

KOLSTRUP, C. L. Work-Related Musculoskeletal Discomfort of Dairy Farmers and Employed Workers. Journal of Occupational Medicine and Toxicology (London, England). 2012; n. 7, p. 23-32.

LEITE, M. R.; ZANETTA, D. M. T.; TREVISAN, I. B.; BURDMANN, E. A.; SANTOS, U. P. Trabalho no Corte de Cana-de-Açúcar, Riscos e Efeitos na Saúde: revisão da literatura. Rev Saúde Pública. 2018; n. 52.

LIGEIRO, J. Ferramentas de Avaliação Ergonômica em Atividades Multifuncionais: a Contribuição da Ergonomia para o Design de Ambientes de Trabalho [Dissertação]. Bauru: Universidade Estadual Paulista Júlio De Mesquita. 2010.

LONGEN, W. C.; BARCELOS, L. P.; KARKLE, K. K.; SCHUTZ, F. S.; VALVASSORI, S. S.; VICTOR, E. G. et al. Avaliação da Incapacidade e Qualidade de Vida de Trabalhadores da Produção de Indústrias Cerâmicas. Ver Bras Med Trab. 2018, v. 16, n. 1.

LÓPEZ-ARAGÓN, L.; LÓPEZ-LIRIA, R.; CALLEJÓN-FERRE, Á-J.; GÓMEZGALÁN, M. Applications of the Standardized Nordic Questionnaire: A Review. Sustainability. 2017; v. 9, n. 9, p. 1514. 
LUNNER-KOLSTRUP, C.; SSALI, T. K. Awareness and Need for Knowledge of Health and Safety among Dairy Farmers Interviewed in Uganda. Frontiers in Public Health. 2016; n. 4, p. 137-142.

MANZOLI, S. T.; IGUTI, A. M.; MONTEIRO, I. Condições de Trabalho e Saúde dePlantadores de Verduras de um Município Brasileiro. Dialnet. 2017, n. 30, p. $269-284$.

MARTINS, M. C.; LONGEN, W. C. Atividade Física Comunitária: efeitos sobre a funcionalidade da lombalgia crônica. Revista Brasileira em Promoção da Saúde. 2017, v. 30, n. 4.

MASSELLI, M. R.; FREGONESI, C. E. P; FARIA, C. R. S.; BEZERRA, M. I. S.; JUNGES, D.; NISHIOKA, T. H. Índice Funcional de OswestryApós Cirurgia para Descompressão de Raízes Nervosas. FisioterMov. 2007; n. 20, p. 115-22.

MCMILLAN, M.; TRASK, C.; DOSMAN, J.; HAGEL, L.; PICKETT, W. Team SFICS. Prevalence of Musculoskeletal Disorders Among Saskatchewan Farmers. J Agromedicine. 2015; n. 20, p. 292-301.

MOREIRA, J. P. L.; OLIVEIRA, B. L. C. A; MUZI, C. D.; CUNHA, C. L. F.; BRITO, A. S.; LUIZ, R. R. A Saúde dos Trabalhadores da Atividade Rural no Brasil. Cad. Saúde Pública.2015; 31(8): 1698-1708. National Center for Farmworker Health, Inc. NCFH. Farmworker Occupational Health and Safety. 2013. Nove de Julho; 2009. Disponível em: <https://bibliotecatede.uninove.br/bitstream/ tede/849/1/B_Joanna\%20Carolina\%20Bachi>. Acesso em: 10 jun. 2018.

OSBORNE, A.; BLAKE, C.; FULLEN, B. M.; MEREDITH, D.; PHELAN, J.; MCNAMARA, J. et al. Prevalence of Musculoskeletal Disorders Among Farmers: a systematic review. Am J Ind Med. 2012; n. 55, p. 143-158.

PESONEN, A. Pain Measurement and Management in the Elderly Patients: clinical studies in long term hospital care and after cardiac surgery [dissertação na Internet]. Helsinki, Finland: Department of Anaesthesiology and Intensive Care; 2011. Disponível em: <http://www.jpsmjournal.com/article/S08853924(05)00611-1/pdf>. Acesso em: 15 jun. 2018. 
PRIUlI, R. M. A.; MORAES, M. S.; CHIARAVALlOTI, R. M. Impacto do Estresse na Saúde de Cortadores de Cana. Rev Saúde Pública. 2014, v. 48, n. 2, p. 255-231.

ROCHA, L. P.; CEZAR-VAZ, M. R.; DE ALMEIDA, M. C. V.; PIEXAK, D. R.; BONOW, C. A. Associação entre a Carga de Trabalho Agrícola e as Dores Relacionadas. Acta Paulista de Enfermagem. 2014; n. 27, p. 4.

SANTOS, G. E. O. Cálculo Amostral: calculadora on-line. 2018.

SARAH, G. D.; HUDSON, S.; JEAN, E.; HAY-SMITH, C.; MILOSAVLJEVIC, S. Rural Workers' Experience of Low Back Pain: exploring why they continue to work. J Occup Rehabil. 2011; n. 21(3), p. 395-409.

SILVA, S. H.; VASCONCELOS, A. G.; GRIEP, R. H.; ROTENBERG, L. TestRetest Reliability of the Work Ability Index (WAI) in Nursing Workers. Ver Bras Epidemiol. 2013; n. 16(1), p. 202-9.

SOUZA, K. R.; MENDONÇA, A. L. O.; RODRIGUES, A. M. S.; FELIX, A. G.; TEIXEIRA, L. R.; SANTOS, M. B.; MOURA, M. A Nova Organização do Trabalho na Universidade Pública: consequências coletivas da precarização na saúde dos docentes. Ciência \& Saúde Coletiva. 2017, n. 22(11).

STADLER, S. T.; RIBEIRO, V. V.; FRANÇA, D. M. V. Z. Autopercepção de Dificuldade Auditiva, Hábitos e Fatores de Risco para Perda Auditiva em Agricultores. Rev CEFAC. 2016; n. 18(6).

TAGHAVI, S. M.; MOKARAMI, H.; AHMADI, O.; STALLONES, L.; ABBASPOUR, A.; MARIORYAD, H. Risk Factors for Developing WorkRelated Musculoskeletal Disorders during Dairy Farming. The international journal of occupational and environmental medicine. 2017 ; n. 8, p. 39-45.

TECKLE, P.; HANNAFORD, P.; SUTTON, M. Is the Health of People Living in Rural areas Different from Those in Cities? Evidence from routine data linked with the Scottish Health Survey. BMC Health Services Research. 2012; n. 12, p. 43. 
VILLAREJO, D.; MCCURDY, S. A.; BADE, B.; SAMUELS, S.; LIGHTHALL, D.; WILLIAMS, D. The Health of California's Immigrant Hired Farm workers. Am J Ind Med. 2010; n. 53(4), p. 387-97.

WALKER-BONE, K.; PALMER, K. Musculoskeletal Disorders in Farmers and Farm Workers. Occup Med (Lond) 2002; n. 52, p. 441-50.

WALSH, I. A. P. Aspectos Clínicos e Funcionais em Trabalhadores Ativos com e sem Sintomas e Evidências de DORT [Tese]. São Carlos: Universidade Federal de São Carlos. 2005. 